\title{
EFEITO DE DIFERENTES INTENSIDADES DE LUZ SOBRE A RESPOSTA AO ESTRESSE, CRESCIMENTO E ÍNDICE REPRODUTIVO EM FÊMEAS DE LAMBARI (Astyanax bimaculatus)
}

\author{
NAVARRO, Fernanda Keley Silva Pereira ${ }^{1}$ \\ NAVARRO, Rodrigo Diana ${ }^{1}$ \\ MURGAS, Luiz David Solis ${ }^{2}$
}

\begin{abstract}
RESUMO: Este trabalho teve como objetivo verificar o efeito de diferentes intensidades de luz sobre a resposta ao estresse, crescimento e maturação ovariana em fêmeas de lambari (Astyanax bimaculatus). Vinte juvenis de lambari foram mantidos em aquários de 20L cada um, em delineamento inteiramente ao acaso, com intensidade luminosa de 1173lux, provida de uma lâmpada fluorescente. Outras 20 fêmeas de lambaris foram distribuídas em uma apa situada em um tanque retangular de alvenaria, com intensidade de luz natural de 890lux. Após 40 dias de experimento os peixes, mantidos a jejum de 24 horas, foram previamente anestesiados com benzocaína. Dados de peso final, peso da gônada, comprimento total e índice gonadossomático foram mensurados. Amostras de sangue, através de punção caudal, foram coletadas para as dosagens de glicose e cortisol. Em relação aos resultados, não foram observadas diferenças significativas para níveis plasmáticos de cortisol e glicose entre os tratamentos, porém a taxa de sobrevivência do tratamento com 1173lux foi significativamente menor em relação ao tratamento com 890lux. Os peixes do tratamento com 890lux de intensidade luminosa apresentaram, significativamente, maior peso final, maior peso de gônada e maior IGS em relação ao tratamento de 1173lux. Para comprimento total, não foi observado diferença significativa. Os resultados obtidos neste estudo permitem concluir que a intensidade de luz desempenha um importante papel no bem-estar e na maturação ovariana de fêmeas de lambaris.
\end{abstract}

Palavras-chave: Lambari. Intensidade de luz. Estresse. Índice gonadossomático.

\section{EFFECT OF DIFFERENT LIGHT INTENSITIES ON THE STRESS RESPONSE, GROWTH AND OVARIAN MATURATION IN FEMALE OF LAMBARI (Astyanax bimaculatus)}

SUMMARY: This study aimed to evaluate the effect of different intensities of light on the stress response, growth and ovarian maturation in female of lambari (Astyanax bimaculatus). Twenty juveniles were kept in aquariums tetra $20 \mathrm{~L}$ each, in a completely randomized design, with light intensity of 1173 lux fitted with a fluorescent lamp. Another 20 females were distributed in a tetra apa located in a rectangular tank of masonry with light intensity of natural 890lux. After 40 days of the experiment the fish kept on fasting for 24 hours, were previously anesthetized. Data from the final weight, gonad weight, total length and gonadosomatic index were measured. Samples of blood, through caudal puncture, were collected for assay of glucose and cortisol. In the results, there were no significant differences in plasma levels of cortisol and glucose between treatments, but the survival rate of treatment with 1173lux was significantly lower compared to treatment with 890lux. Fish treatment with 890lux light intensity had significantly higher final weight, weight of gonad higher and higher GSI in relation to the treatment of 1173lux. For total length, there was no significant difference. The results of this study indicate that the intensity of light plays an important role in welfare and ovarian maturation of female of lambaris. Furthermore, data suggest that fish subjected to light intensity of 1173lux are more stressed and aggressive towards 890lux regime, whose females had a better ovarian development.

Keyworks: Lambari, Light intensity. Stress. Gonadosomatic index.

\footnotetext{
${ }^{1}$ Grupo de pesquisa em aquicultura AcquaUnB da Universidade de Brasília, Campus Universitário Darcy Ribeiro, Caixa Postal 4.508, CEP 70910-970 Brasília, DF. E-mail: fbionavarro@gmail.com, navarrounb@gmail.com

${ }^{2}$ Setor de Fisiologia e Farmacologia do Departamento Medicina Veterinária da Universidade Federal de Lavras, Departamento de Medicina Veterinária, CEP: 37200-00 Lavras, MG. E-mail: 1smurgas@ ufla.br
} 


\section{INTRODUÇÃO}

O Brasil reúne condições extremamente favoráveis à piscicultura. Além do grande potencial de mercado, o país conta com clima favorável, boa disponibilidade de áreas, grandes safras de grãos (soja, milho, trigo, entre outros que geram matérias primas para rações animais) e invejável potencial hídrico (BOZANO, 2002). Entre as espécies mais cultivadas, estão as de origem exóticas, como a carpa (Cyprinus carpio) e a tilápia (Oreochromis niloticus). Entretanto, dentro da ampla diversidade da fauna aquática brasileira, são encontradas espécies que se adéquam perfeitamente ao cultivo, como o pacu, o tambaqui, o piavuçu, lambari, entre outros.

O lambari Astyanax bimaculatus é uma espécie nativa do Brasil, pertencente à família Characidae, que possui distribuição desde o Nordeste brasileiro até a bacia do Prata (Barbieri et al., 1982). Esta espécie, de pequeno porte, aceita alimentação artificial com bastante facilidade e apresenta bom potencial para a aqüicultura, podendo ser utilizados como iscas em pesca esportivas, como petiscos e como peixes ornamentais (SATO et al., 2006). Além disso, os lambaris possuem grande importância ecológica ao fazerem parte da dieta de vários vertebrados e serem dispersores secundários de sementes auxiliando na preservação das matas ciliares (FERNANDES; MARTINS-SANTOS, 2004).

O efeito de variáveis ambientais sobre o crescimento, sobrevivência e as respostas fisiológicas dos peixes têm sido extremamente estudadas. Alguns estudos e investimentos xcientíficos vêm sendo feitos na área de piscicultura a fim de minimizar o estresse dos peixes decorrente de diversos fatores como manejo, estado nutricional, qualidade da água, temperatura, fotoperíodo, salinidade (BANI et al., 2009, NAVARRO; NAVARRO, 2012).

O cortisol, o principal glicocorticóide sintetizado no tecido interrenal de peixes, possui um importante papel na resposta ao estresse, comportamento, osmorregulação, metabolismo, crescimento, reprodução e função imune (WENDELAAR BONGA, 1997). Em peixes teleósteos, a elevação plasmática de cortisol e glicose são reconhecidas como as principais respostas ao estresse, sendo amplamente utilizado como indicadores dessa resposta $\mathrm{O}$ cortisol é utilizado para caracterizar a resposta primária, e a glicose a resposta secundária do estresse em peixes (NAVARRO; NAVARRO, 2012).

A diversidade de repostas a luz entre os peixes pode ser reflexo de adaptações específicas ao seu ambiente, onde a luz pode variar em termos de quantidade (intensidade), qualidade (espectro) e duração (fotoperíodo) (BANI et al., 2009, NAVARRO; NAVARRO, 2012).

A influência de fotoperíodo e de níveis de luminosidade na larvicultura e alevinagem confinada de espécies nativas tem sido pouquíssimo estudada no Brasil, até porque quase todas as estações de produção de alevinos utilizam tanques externos (Behr et al., 1999). A intensidade de luz é propensa a variações diárias, dependendo da hora do dia (madrugada e no crepúsculo), das condições meteorológicas ou da fase da lua (FÁLCON, et al., 2010). A maioria dos peixes utiliza a visão para se alimentar e precisam de um limiar mínimo de intensidade de luz para ser capaz de se desenvolver e crescer normalmente (BLAXTER, 1986; OUNAIS-GUSCHEMANN, 1989; BOEUF; LE BAIL, 1989). A influência da intensidade luminosa em peixes pode variar com a espécie e com estágio de desenvolvimento (BOEUF; LE BAIL, 1989). Alta intensidade de luz pode ser estressante ou até mesmo letal (BOEUF; LE BAIL, 1989).

O efeito da intensidade de luz na sobrevivência e crescimento de larvas ou juvenis tem sido estudada em larvas de peixes chatos (BLAXTER, 1986), larvas de bacalhau (HUSE, 1994), larvas do bacalhau do Atlântico (PUVANENDRAN; BROWN, 2000), pós-larvas de robalo (CUVIER; PÉRES et al., 2001) juvenis de arinca (TRIPPEL; NEIL, 2003). A influência da intensidade de luz sobre a atividade de natação e alimentação (PETRELL; ANG, 2001; ALMAZA'N-RUEDA et al., 2004), o canibalismo 
(HECHT; PIENAAR, 1993; GARDNER; MAGUIRE, 1998; KESTEMONT et al.., 2003), hormônios fisiológicos (BOEUF; LE BAIL, 1989) e metabolismo (APPELBAUM; KAMLER, 2000) também foram relatados.

Estudos que visem esclarecer as influências da intensidade luminosa sobre o desenvolvimento, bem-estar e padrões fisiológicos de espécies nativas do Brasil são escassos na literatura. Assim, objetivouse avaliar o efeito de diferentes intensidades de luz sobre a resposta ao estresse, crescimento e maturação ovariana em fêmeas de lambari (Astyanax bimaculatus).

\section{MATERIAL E MÉTODOS}

Os experimentos foram conduzidos no laboratório de Fisiologia e Farmacologia, do Departamento de Medicina Veterinária da Universidade Federal de Lavras - UFLA e na Estação de Piscicultura do Departamento Zootecnia, da Universidade Federal de lavras - UFLA entre o período de 25 de janeiro a 07 de março de 2010, com duração de 40 dias.

Foram utilizados 40 fêmeas de lambari, Astyanax bimaculatus, provenientes da estação de piscicultura da Universidade Federal de Viçosa (UFV), com peso de $7.2 \pm 2.4 \mathrm{~g}$ e comprimento de 7,5 \pm $0.8 \mathrm{~cm}$. Após 15 dias de aclimatação, 20 peixes foram acondicionados e distribuídos em 2 aquários de 20 litros cada um, em delineamento inteiramente ao acaso, em sistema fechado de recirculação de água e biofiltro. Os outros 20 peixes foram distribuídos em uma apa situada em um tanque retangular de alvenaria. Cada peixe foi considerado uma unidade experimental. Os tratamentos diferenciaram na densidade de estocagem e na intensidade luminosa.

O controle das horas de luz fornecidas durante o experimento em laboratório foi feito por "timers" automáticos que apagaram e acenderam as luzes fluorescentes das bancadas durante os períodos estipulados, e a intensidade luminosa foi de 1173 lux no período de luz, durante um fotoperíodp de 12Luz:12Escuro. Durante o experimento na piscicultura a intensidade de luz natural foi de 890 lux na fase clara, durante um fotoperíodo tropical, que segundo Fálcon et al. (2010) prevalece em torno de 12L:12E durante todo ano.

Foram mensurados os parâmetros de qualidade de água: oxigênio dissolvido, $\mathrm{pH}$, temperatura, diariamente. Os níveis de oxigenação foram mantidos com auxílio de aeradores, e mensurados através de um oxímetro (YSI, USA), o pH foi medido através do peagâmetro e a temperatura com o uso de termômetro digital.

Durante o manejo alimentar, foi utilizado uma ração comercial contendo $28 \%$ de PB e $3.100 \mathrm{kcal}$ de ED/kg (Tropical NUTRON Hi-Fi). A quantidade de ração fornecida, diariamente, foi de $2 \%$ do peso vivo e foi dividida em duas refeições diárias; a primeira ração foi fornecida às 9 horas e a segunda às 16 horas. Para o experimento em laboratório, após 15 minutos do manejo alimentar, os aquários foram sinfonados para a retirada das sobras de ração.

Procedimentos de coleta e Análises bioquímicas

Ao término do experimento, foram feitas coletas de sangue para mensuração de glicose sanguínea, com o objetivo de verificar indicadores de estresse nos peixes e diferenças causadas pelos diferentes fotoperíodos. Exemplares de peixes submetidos a jejum de 24 horas foram previamente anestesiados com uma solução contendo $400 \mathrm{mg} / \mathrm{L}$ de benzocaína para dar início aos procedimentos de coleta. Dados de peso, comprimento total referente a cada peixe dos diferentes tratamentos foram mensurados por meio de balança de precisão $(0.001 \mathrm{~g})$. Foram utilizados 12 peixes de cada tratamento, sob jejum de 24 
horas, para as coletas de amostras sanguíneas através de punção caudal, utilizando-se seringas de $1 \mathrm{~mL}$.

Uma pequena parte do sangue coletado foi utilizada para quantificar a glicose sanguínea através de aparelho digital de glicose: ACCU - CHEC SOFTCLICK - ESTADOS UNIDOS. A maior parte do sangue coletado foi depositada em eppendorfs contendo heparina e deixada em gelo até que as amostras de todos os peixes fossem coletadas. O sangue coletado foi centrifugado a $5000 \mathrm{rpm}$ por 10 minutos. Em seguida, o plasma heparinizado (sobrenadante) foi retirado com uma pipeta digital. O plasma coletado foi armazenado em eppendorfs e etiquetados para posteriores análises hormonais. Todo material plasmático foi congelado a $-20^{\circ} \mathrm{C}$ até o momento da análise.

Para determinação do cortisol, o plasma foi analisado através da técnica ELISA (Kit CORTISOL 96T. ELISA - HUMAN).

As análises estatísticas foram realizadas através do programa SAS, sendo as médias comparadas pelo teste $\mathrm{t}$ com $5 \%$ de significância.

\section{RESULTADOS E DISCUSSÃO}

Durante o período experimental, valores médios obtidos de temperatura média de $28,67 \pm 0,73 ; 6,65$ $\pm 0,68$ para $\mathrm{pH} ; 5,23 \pm 0,85 \mathrm{mg}$ / L para oxigênio dissolvido, estiveram dentro das faixas consideradas aceitáveis para o cultivo de peixes.

Após os 40 dias de experimento, não foram observadas diferenças significativas para níveis plasmáticos de cortisol e glicose entre os tratamentos, porém a taxa de sobrevivência do tratamento com 1173lux foi significativamente menor em relação ao tratamento com 890lux.

Tabela 1. Concentração plasmática de glicose, cortisol e taxa de sobrevivência de fêmeas de lambaris em função da intensidade luminosa e densidade de estocagem.

\begin{tabular}{ccc}
\hline Tratamento & 1173 lux & $8901 \mathrm{lux}$ \\
\hline Glicose $(\mathrm{mg} / \mathrm{dL})$ & $65,183^{\mathrm{a}}$ & $65,08^{\mathrm{a}}$ \\
Cortisol $(\mathrm{ng} / \mathrm{mL})$ & $190,00^{\mathrm{a}}$ & $171,33^{\mathrm{a}}$ \\
Taxa de Sobrevivência $(\%)$ & $30^{\mathrm{a}}$ & $100^{\mathrm{b}}$
\end{tabular}

Médias na mesma linha com diferentes sobrescritos são significantemente diferentes segundo teste $\mathrm{t}(\mathrm{P}<0,05)$.

No estudo de Correia (2008), com lambaris da espécie Astyanax fasciatus, foi verificado níveis basais de cortisol na faixa de $35 \mathrm{ng} / \mathrm{mL}$. Este estudo sugere que as fêmeas de lambari (Astyanax bimaculatus) do presente estudo mostraram níveis elevados tanto de cortisol quanto de glicose para ambos os tratamentos (tabela 1). Provavelmente, o elevado nível de cortisol levou a um aumento nos níveis plasmáticos de glicose. O cortisol, hormônio provido da glândula interrenal, estimula a glicogenólise e a gliconeogênese em peixes (MARTÍNEZ-PORCHAS et al., 2009). Este hormônio é utilizado para caracterizar a resposta primária, e a glicose a resposta secundária do estresse em peixes (ADAMANTE, 2005). Além disso, a baixa taxa de sobrevivência somado aos sinais de agressividade (feridas no corpo) dos animais submetidos à intensidade de luz de 1173lux suporta a idéia desta faixa de luminosidade ter gerado estresse nestes peixes, o que não foi observado nos peixes sob regime de 890lux (tabela 1).

A maioria das pesquisas sobre os efeitos da intensidade de luz tem-se concentrado em larvas, poucos são os estudos com juvenis de peixes. Os resultados do presente estudo corroboram com os estudos de Appelbaum e Kamler (2000) que verificaram que uma maior intensidade da luz exerce um 
efeito indireto sobre a mortalidade de juvenis de bagre Africano, aumentando a atividade locomotora e o canibalismo. Almaza'n-Rueda et al. (2004) também observaram em Clarias gariepinus um aumento da atividade natatória e maior agressividade entre os peixes submetidos a 150lux em relação a um regime de 15lux. No entanto, na pesquisa de Han et al. (2005), com juvenis de Leiocassis longirostris os peixes apresentaram menor crescimento e taxa de sobrevivência para o tratamento com menor intensidade luminosa (5lux).

Além disso, a manipulação da intensidade luminosa pode afetar a síntese e liberação de outros hormônios como melatonina, hormônio da glândula pineal, liberado em maior quantidade em baixa intensidade luminosa ou durante a ausência de luz (EKSTRÖM; MEISSL, 1997; BAYARRI et al., 2002). MUNRO (1986) observou no ciclídeo Aequidens pulcher, o papel deste hormônio na redução de respostas agressivas. Alguns estudos com trutas e salmãos do Atlântico expostos ao período noturno, verificaram que o aumento da intensidade de luz gera uma diminuição dos níveis de melatonina (ZACHMANN et al., 1992; PORTER et al., 2001).

Os peixes do tratamento com 890lux de intensidade luminosa apresentaram, significativamente $(\mathrm{p}<0,05)$, maior peso final, maior peso de gônada e maior IGS em relação ao tratamento de 1173lux (tabela 2). Para comprimento total, não foi observado diferença significativa (tabela 2). Nos peixes, o estresse provoca efeitos adversos sobre o desempenho reprodutivo de fêmeas. Dependendo do período e da intensidade do agente estressor, este pode provocar atresia folicular, oócitos avanço ou atraso na maturação e ovulação ou afetar o tamanho do ovo, o sucesso da fertilização (CLEARWATER; PANKURST, 1997; SCHRECK et al., 2001). O estresse gerado nas fêmeas submetidas à intensidade luminosa de 1173lux, possivelmente, levou a um menor desenvolvimento ovariano destes peixes.

Apesar dos elevados níveis de cortisol e glicose, as fêmeas de lambari sob regime de 890lux mostraram um melhor avanço em relação a maturação ovariana. Provavelmente, a elevada concentração plasmática de glicose deve-se a ação metabólica do cortisol em estimular a produção deste substrato a fim de atender a demanda energética durante a fase reprodutiva. Além do papel do cortisol nas respostas ao estresse e inflamatórias, no metabolismo energético e na ormorregulação de peixes, a função deste hormônio na reprodução destes animais vêm sendo bastante investigada (MILLA et al., 2009). A elevada concentração plasmática de corticosteróides, inclusive de cortisol em fêmeas maduras de diversos teleósteos, foi relatado por diversos autores (MILLA et al., 2009). Algumas pesquisas demostraram o papel do cortisol na indução da primeira maturação sexual, na indução da maturação meiótica dos oócitos, na maturação dos oócitos em sinergismo ou não com o MIS (hormônio indutor da maturação) além de sua participação no processo de hidratação dos oócitos (SKJAERAASE et al., 2004; FISZBEIN et al., 2010). Logo, os dados reprodutivos das fêmeas de lambari sob regime de 890lux do presente trabalho (peso gônada, IGS), juntamente com os achados anteriores referentes a importância do cortisol na reprodução, sugerem que a elevação dos níveis de cortisol deve-se ao estágio avançado de maturação gonadal ao qual os peixes se encontraram ao final do experimento.

Tabela 2. Peso final $(\mathrm{g})$, comprimento total $(\mathrm{cm})$ de fêmeas de lambaris em função do fotoperíodo.

\begin{tabular}{ccc}
\hline Tratamento & $11731 \mathrm{lux}$ & $8901 \mathrm{ux}$ \\
\hline Peso final (g) & $7,017^{\mathrm{a}}$ & $9,98^{\mathrm{b}}$ \\
Comprimento total (cm) & $8,100^{\mathrm{a}}$ & $8,53^{\mathrm{a}}$ \\
Peso da gônada (g) & $0,86^{\mathrm{a}}$ & $1,71^{\mathrm{b}}$ \\
Índice Gonadossomático (IGS) & $12,26^{\mathrm{b}}$ & $17,13^{\mathrm{a}}$
\end{tabular}

Médias na mesma linha com diferentes sobrescritos são significantemente diferentes segundo teste $\mathrm{t}(\mathrm{P}<0,05)$. 


\section{CONCLUSÃO}

Os resultados obtidos neste estudo permitem concluir que a intensidade de luz desempenha um importante papel no bem-estar e na maturação ovariana de fêmeas de lambaris. Além disso, os dados sugerem que os peixes submetidos a intensidade de luz de 1173lux ficam mais estressados e agressivos em relação ao regime de 890lux, cujas fêmeas apresentaram um melhor desenvolvimento ovariano.

\section{REFERÊNCIAS}

ALMAZA N-RUEDA, P.; SCHRAMA, J.W.; VERRETH, J.A.J., Behavioural responses under different feeding methods and light regimes of the African catfish (Clarias gariepinus) juveniles. Aquaculture, Amsterdam, 231, 347 - 359, 2004.

ALVARINO, J.M.; RANDALL, C.F.; BROMAGE, N., 1993. Pattern of melatonin secretion in the rainbow trout exposed to light pulses of different duration and intensity. Actas IV. Congresso Nacional Acuicultura, 191-196.

ADAMANTE, W.B. Estresse de juvenis de dourado e mandi sob diferentes densidades e tempos de transporte. 2005. 39 p. Dissertação (Mestrado em Aquicultura) - Universidade Federal de Santa Catarina, Florianópolis.

APPELBAUM, S.; KAMLER, E; Survival, growth, metabolism and behaviour of Clarias gariepinus (Burchell 1822) early stages under different light conditions. Aquacultural Engineering, Seattle, v.22, p.269-287, 2000.

BANI,A.et al. Effects of different photoperiods on growth, stress and haematological parameters in juvenile great sturgeon Huso huso. Aquaculture Research, Oxford, v. 40,p. 1899-1907, 2009.

BARBIERI, G. Biologia de Astyanax scabripinnis paranae (Characiformes, Characidae) do Ribeirão do Fazzari, São Carlos, São Paulo II. Aspectos quantitativos da reprodução. Revista Brasileira de Biologia, São Carlos, v.52, n.4, p.589-596. 1992.

BAYARRI, M. J.; MADRID, J. A.; SÁNCHEZ-VÁZQUEZ, F. J. Influence of light intensity, spectrum and orientation on sea bass plasma and ocular melatonin. Journal of Pineal Research,Texa, v.32,p. 3440, 2002.

BEHR, E.R.et al. Influência de diferentes níveis de luminosidade sobre o desempenho de larvas de jundiá (Rhamdia quelen) (Quoy e Gaimard, 1824) (Pisces: Pimelodidae). Acta Scientiarum, Maringa v. 21, n. 2, p. 325-330, 1999.

BLAXTER, J.H.S. Pattern and variety in development. In: HOAR, W.S.; RANDALL, D.J. (Eds.) Fish physiology. San Diego: Academic Press, 1988. p.1-48.

BOEUF, G.; Le BAIL, P.Y., Does light have an influence on fish growth? Aquaculture, Amsterdam, v. 177, p. $129-152,1989$.

BOZANO, G. L. N. Viabilidade Técnica da Produção de Peixes em Tanques-rede. In: Simpósio Brasileiro de Aqüicultura, 12, 2002, Goiânia. Anais... Goiânia: Abraq. p. 107-111, 2002.

CORREA, T. G. Influencia do alumínio e do pH ácido sobre a fisiologia reprodutiva peixes teleósteos continentais. 2008. 204p.Dissertação (Mestrado) Instituto de Biociência da Universidade de São Paulo. 
CLEARWATER, S.J.; PANKHURST, N.W. The response to capture and confinement stress of plasma cortisol, plasma sex steroids and vitellogenic oocytes in the marine teleost, red gurnard. Journal of Fish Biology, London, v.50, p. 429-441, 1997.

CUVIER-PE'RES, A.et al. Effects of light intensity on animal husbandry and digestive enzyme activities in sea bass Dicentrachus labrax post-lavae. Aquaculture, Amsterdam, v. 202, p.317 - 328, 2001.

FÁLCON, J.et al. Current knowledge on the melatonin system in teleost fish. General and Comparative Endocrinology, Denver, v.165, p.469-482, 2010.

FERNANDEZ, P.J.; BAGNARA, J.T.; Effect of background color and low temperature on skin color and circulating alpha-MSH in two species of leopard frog. General and Comparative Endocrinology, Denver, v.83, p.132 - 141, 1991.

FISZBEIN, A.et al. Photoperiodic modulation of reproductive physiology and behaviour in the cichlid fish Cichlasoma dimerus. Physiology \& Behavior, Elmsford, v.99, p.425-432, 2010.

FUTTER, C.W.et al. The effects of differential light intensities on the diel rhythm of melatonin release in rainbow trout. 1999. In: TARANGER, G.L. et al. (ed.), In: INTERNATIONAL SYMPOSIUM ON REPRODUCTIVE PHYSIOLOGY OF FISH, 6 Proceedings.... Bergen, 4-9 jul. 1999, p. 340.

GARDNER, C.; MAGUIRE, G.B.; Effect of photoperiod and light intensity on survival, development and cannibalism of larvae of the Australian giant crab Pseudocarcinus gigas (Lamarck). Aquaculture, Amsterdam 165, p. $51-63,1998$.

HAN, D., XIE, S.;LEI, W., ZHU, X.; YANG, Y., Effect of ration on the growth and energy budget of Chinese longsnout catfish Leiocassis longirostris Gqnther. Aquaculture Reseach, Oxford, v.35, p. 866873, 2004.

HECHT, T.; APPELBAUM, S., Notes on the growth of Israeli sharptooth catfish (Clarias gariepinus) during the primary nursing phase. Aquaculture, Amsterdam, v.63, p. 195 - 204, 1987.

HUSE, I.J. Feeding at different illumination levels in larvae of three marine teleost species: cod, Gadusmorhua L., plaice, Pleuronectes platessa L., and turbot, Scophthalmus maximus (L.). Aquaculture Research, Oxford, v.25, p.687 - 695, 1994.

KESTEMONT, P.et al. Size heterogeneity, cannibalism and competition in cultured predatory fish larvae: biotic and abiotic influences. Aquaculture, Amsterdam, v.227, p.333 - 356, 2003.

MARTÍNEZ-PORCHAS, M.; MARTÍNEZ-CÓRDOVA, L. R.; RAMOS-ENRIQUEZ R. Cortisol and Glucose: Reliable indicators of fish stress? Pan-American Journal of Aquatic Sciences, Dartmouth, v.4, p.158-178, 2009.

MENDONÇA, P.P.et al. Influência do fotoperíodo no desenvolvimento de juvenis de tambaqui (Colossoma Macropomum). Archivos de Zootecnia.Cordoba, v.58,p.323-331. 2009.

MILLA S.et al. Ovarian steroidogenesis inhibition by constant photothermal conditions is caused by a lack of gonadotropin stimulation in Eurasian perch. General Comparative Endocrinology, Denver, v.163, p.242-250, 2009.

MUNRO, A. D. Effects of melatonin, serotonin, and naloxone on aggression in isolated cichlid fish (Aequidens pulcher). Journal of Pineal Research, Texas, 3, 257-262. 1986.

NAVARRO, F.K.S.P.; NAVARRO,R.D.. Importância do fotoperíodo no crescimento e na reprodução de peixes. Revista Brasileira Reprodução Animal, Belo Horizonte, v.36,p.94-99. 2012 
OUNAIS-GUSCHEMANN, N., De'finition d'un mode'le d'e'levage larvaire intensif pour la daurade, Sparus auratus. The`se de doctorat de l'Universite' d'Aix-Marseille II, 184 pp.1989.

PORTER, M.J.R.; DUNCAN, N.; STEFANSSON, S.O.; BROMAGE, N.R.; Temperature, light intensity and plasma melatonin levels in juvenile Atlantic salmon. Journal Fish Biology, London, 58, p. 431-438. 2001.

PUVANENDRAN, V.; BROWN, J.J.; Effect of light intensity on the foraging and growth of Atlantic cod larvae: interpopulation difference? Marine Ecology Progress, v.167, p. 207 - 214. 2000.

PETRELL, R.J.;ANG, K.P., Effects of pellet contrast and light intensity on salmonid feeding behaviours. Aquacultural Engineering, Seattle, v.25, p.175 - 186, 2001.

SATO, Y.et al. Biologia reprodutiva e reprodução induzida de duas espécies de characidae (Osteichthyes, Characiformes) da bacia do São Francisco, Minas Gerais, Brasil. Revista Brasileira de Zoologia, Curitiba, v.23, p.267-273, 2006.

SCHRECK, B.;CONTRERAS-SANCHEZ, W.; FITZPATRICK, M., Effects of stress on fish reproduction, gamete quality, and progeny. Aquaculture, Amsterdam, v.197, p.3 - 24, 2001.

SKJAERAASEN, J.E.et al. The effect of photoperiod on sexual maturation, appetite and growth in wild Atlantic cod (Gadus morhua). Fish Physiology and Biochemistry, Netherlands v.30, p.163-174, 2004.

TRIPPEL, E.A.; NEIL, S.R.E., Effects of photoperiod and light intensity on growth and activity of juvenile haddock (Melanogrammus aeglefinus). Aquaculture, Amsterdam, v.217, p. 633 - 645, 2003.

WENDELAAR BONGA,S.E. The stress response in fish. Physiological Reviews, Bethesda v. 77, p.591625, 1997.

ZACHMANN, A.et al. Effects of photoperiod and temperature on rhythmic melatonin secretion from the pineal organ of the white sucker ŽCatostomus commersoni. In vitro. General and

Comparative Endocrinology, Denver, v.86, p.26-33, 1992. 